\title{
SOBRE LA NATURALEZA DEL LENGUAJE
}

\author{
Jack L. Wilson
}

En nuestro medio está bastante arraigada la idea de que todo lenguaje es comunicación, y, a la inversa, hay quien supone que toda comunicación animal o humana es lenguaje. Se oye hablar, por ejemplo, de "la lengua escrita, el lenguaje de los gestos, el lenguaje de las abejas, de las loras, o de este animal o del otro'. Pero, más que comunicación, podríamos definir el lenguaje como un sistema de simbolización que media, en una forma sumamente compleja, entre el universo del significado y el universo del sonido.

En los animales hay comunicación. En el llamado 'lenguaje de las abejas', se supone que la abeja que encuentra una fuente de néctar vuelve a su colmena donde procede a comunicar a sus compañeros la dirección y la distancia a que está esta fuente mediante una danza. Hay una danza para expresar que la fuente está a menos de cien metros de la colmena, y otra, más agitada, cuando está a más distancia. La velocidad de la danza indica la distancia desde la colmena, y el lugar en la superficie de la colmena donde se efectúa indica la dirección que deben seguir las otras abejas. A esta clase de sistema estructurado de comunicación se le ha llamado 'sistema continuo' porque hay un continuo de posibles direcciones y hay un continuo de posibles distancias. Es un sistema más complejo que el simple emparejamiento de un solo símbolo con un solo referente. La intensidad de la danza tiene un significado más allá del mero hecho de la danza.

No sólo las abejas usan un sistema continuo de comunicación, sino también los primates, entre ellos el hombre, especialmente para la comunicación de grados de emoción. Una mujer asustada, por ejemplo, puede lanzar un grito de más intensidad, de más duración y en un tono más agudo de acuerdo con el miedo que siente. Pero nada de esto es lenguaje.

El otro tipo de sistema estructurado de comunicación se llama 'sistema discreto' y es el sistema representado en su forma más simple por el silbido de la sirena de una fábrica para indicar que es mediodía. En este sistema un solo concepto, 'mediodía', es expresado mediante un solo símbolo, el silbido. Parece ser que los primates no humanos tienen este sistema y algunos tienen hasta tres docenas de unidades conceptuales discretas en su repertorio. Al mono vervet -del este y del sur de Africa, y parecido al mono verde se le distinguen 36 simbolizaciones, todas acompañadas de gestos. Entre ellos:

alguna amaneza: un ladrido

la no agresión: un besuqueo

viene un grupo extraño: aarr sostenido cercanía de un grupo extraño: aarr breve peligro inminente: rraup 
Digno de mencionar es el hecho de que ni el mono vervet ni los otros primates inteligentes no humanos han demostrado la capacidad de reaccionar ante una situación nueva, o parcialmente nueva, con un llamado diferente que sea el resultado de la combinación de llamados o partes de llamados de los que ya existen.

Si bien este sistema de simbolización es primitivo, no sería inverosímil suponer que nuestros ancestros muy remotos lo poseían, mucho antes de que poseyeran lo que en la actualidad llamamos lenguaje. De hecho el hombre todavía utiliza el sistema. Pero hay dos cosas que tiene el lenguaje que lo separan definitivamente de este sistema primitivo: contiene un número de unidades conceptuales prácticamente ilimitado, más grande que el que contiene cualquier otro sistema natural de comunicación, y constantemente cambia. Parece ser que en un pasado remoto el universo conceptual de nuestros antepasados comenzó a expandirse y siguió expandiéndose, no se sabe cómo ni por qué.

Los mensajes que continuaron comunicando los otros primates están circunscritos de manera sumamente estecha. La información que se trasmite a otro primate que la recibe se refiere principalmente a la actual disposición emocional del que lo transmite. Las consecuencias de una recepción eficaz son principalmente modificaciones de las disposiciones al área de sobrevivencia biológica, por ejemplo la alimentación y la reproducción. Aun sin saber ni cómo ni por qué, es evidente que la evolución del hombre ha incluído como uno de sus componentes más significativos un ensanchamiento extraordinario de su universo conceptual y con éste un tremendo incremento en el número $\mathrm{y}$ en la variedad de ideas que puede comunicar. En la evolución del hombre se nota no solamente un incremento en el número y en la complejidad de las ideas que puede comunicar, sino también un cambio cualitativo. A través del lenguaje el hombre comunica no solamente las emociones y los mensajes que son esenciales para su sobrevivencia, sino también una casi infinidad de estados relacionados, objetos y eventos en los cuales puede o no estar directamente involucrado.

Tal vez lo más asombroso de todo es que el hombre puede comunicar sobre asun- tos totalmente extraños a su medio inmediato -la guerra del 56, las noticias de ayer, el clima de mañana- y hasta sobre asuntos que carecen de realidad por completo: duendes, unicornios, la llorona, etc.

Si el universo conceptual del hombre aumentó en forma prácticamente infinita, no sucedió así con las simbolizaciones que tenía a su disposición para expresarlo. Mientras que en los sistemas primitivos - como en el caso de los primates superiores, con excepción del hombre - cada concepto se expresa a través de un solo símbolo de modo que hay una relación de uno a uno, en el lenguaje humano la infinidad de conceptos no va acompañada de una correspondencia infinita de símbolos, ni podría hacerlo. El hombre dispone solamente de unas cuantas docenas de sonidos elementales, en todos los idiomas, para expresar sus ideas, y es mediante las diferentes combinaciones de estos sonidos que transmite sus ideas. Igual sucede con los recursos gramaticales de que dispone el hombre en todos los idiomas, incluso tomándolos en su conjunto. A diferencia de los animales, el hombre ha aprendido a hacer combinaciones arbitrarias de unidades elementales para expresar la casi infinidad de ideas que es capaz de formar. La teoría lingüística más popular en estos momentos la de Noam Chomsky - se basa en la idea de que una abstracción de estas unidades elementales se transmite genéticamente, así como la capacidad de hacer combinaciones arbitrarias de estas unidades.

De acuerdo con la teoría de Chomsky, una parte del lenguaje es aprendida pero hay otra parte que es innata. Pero ningún animal ha dado muestras de poder aprender más que una mínima parte de lo que aprende el hombre, en cuanto a lenguaje se refiere, y ningún animal ha demostrado poseer ni la más mínima parte de lo que en el hombre se postula como innato.

Entre 1932 y 1968 se habían hecho cinco publicaciones de. experimentos llevados a cabo por psicólogos en los cuales se observaban y se medía las reacciones de chimpancés criados en un ambiente familiar. En tres de estos experimentos los chimpancés fueron criados en familias con niños de la misma edad que sirvieron como grupos de control. De los cinco estudios, cuatro se 
realizaron en los Estados Unidos y uno en la Unión Soviética. Hasta donde fuera posible en estos experimentos, se les trató a los chimpancés como si fueran humanos. Se demostró mediante mediciones de su sensibilidad auditiva que el chimpancé tiene un excelente oído. Sobre el desarrollo cerebral del chimpancé comparado con el del hombre poco se sabe. En un ambiente familiar el chimpancé recién nacido tiene reacciones muy parecidas a las de los humanos recién nacidos. Se adapta rápidamente a la parte física de su medio, muestra un fuerte cariño hacia su tutor o madre experimental, imita varios actos realizados por los humanos adultos que no requieran un entrenamiento especial, y realiza bien muchos de los tests de desarrollo que se han diseñado para niños de edad preescolar, llegando algunas veces incluso a superar al niño.

Hasta los tres años, aproximadamente, la 'edad mental' del chimpancé no está muy atrasada de la edad mental del niño de la misma edad. Al mismo tiempo su desarrollo esqueletal y muscular es mucho más rápido que el del niño. Pero a pesar de su alto nivel de conducta imitativa, nunca trata de copiar o de reproducir sonidos humanos. En esto se diferencia de la lora y de otros pájaros que aprenden a reproducir palabras humanas, o humanoides, pero que son menos aptos en cuanto a la imitación de otros aspectos que no sean los orales.

En varias oportunidades se ha intentado enseñar palabras a chimpancés. En 1909 Witmer logró enseñar la palabra "mama”. El chimpancé pronunciaba bien la 'm', pero la 'a' era sorda. Unos años más tarde Furness logró enseñar a un orangután a decir 'papa' y 'cup'. Más tarde, los señores Hayes lograron enseñar a su chimpancé a decir 'papa, mama' y 'cup' y cuando el chimpancé murió estaba aprendiendo a pronunciar 'up'. Pronunciaba todas estas palabras con el mismo sonido vocálico. Estas palabras, 'mama, papa, cup' y posiblemente 'up' representan el sumum de lo que ha podido hacer un primate al tratar de imitar el habla humana, $\mathrm{y}$ en ningún caso mostraba el animal que relacionaba la palabra que pronunciaba con el objeto a que hacía referencia. Sin duda los centros neurales del cerebro relacionados con el habla son deficientes y es posible, aunque no seguro, que los órganos fonadores sean incapaces de producir los patrones complejos de sonidos que caracterizan al lenguaje humano.

Actualmente, hay dos teorías lingüísticas que están compitiendo: la mecanicista o empirista, y la racionalista. Básicamente la controversia gira alrededor de la naturaleza misma del hombre. De acuerdo con las dos tesis, los animales, excluyendo al hombre, son como las máquinas en el sentido de que dada unaprogramación adecuada su conducta será predecible. En el caso de los animales esta programación toma la forma de algún estímulo. En ambas teorías se ha notado que cualquier cosa que pudiéramos llamar 'comunicación animal' es el resultado de algún estímulo y que ese estímulo es inmediato. Las abejas, por ejemplo, no pueden comunicar nada con respecto a alguna fuente de néctar que pudieran haber descubierto el día anterior. Ni los primates no humanos más inteligentes han dado muestras de poder comunicar nada que no se relacione con el presente o el pasado inmediato.

Pero en cuanto al hombre, las dos teorías difieren. De acuerdo con la teoría mecanicista, la conducta (behavior) del hombre, en todo sentido y al igual que los animales, está bajo el control de uno o de varios estímulos; es decir, el hombre también es una especie de máquina no importa cuán complicada o cuán superior sea. De acuerdo con esta teoría, el niño nace con una capacidad que es transmitida genéticamente, pero esta capacidad es parte de su conducta intelectual general. Su mente es como un pizarrón en que nada se ha escrito y que se va llenando conforme el niño va aprendiendo. Los primeros actos conscientes de comunicación se producen cuando el niño se da cuenta de que por ciertos actos suyos se produce alguna reacción, como por ejemplo si llora alguien le trae el biberón de leche. Antes había actuado, llorando, debido al estímulo que le producía el hambre. El niño pronto comienza a tratar de imitar lo que oye a su alrededor, si bien al principio su única producción es un balbuceo, pero en relativamente poco tiempo aprende a hacer distinciones de los sonidos de modo que se le 
entienda. A la imitación le sigue un proceso de aprendizaje por analogía, y con este proceso se completa la tarea de aprender a hablar la lengua materna. El proceso es más o menos así: el niño oye y aprende a imitar oraciones como las siguientes:

'Su papá quiere una naranja. Déle a su papá una naranja'.

'Su mamá quiere una naranja. Déle a su mamá una naranja'.

'Su hermanita quiere una naranja. Déle a su hermanita una naranja'. ' $\mathrm{Su}$ hermanito quiere una naranja. Déle

Para todas las oraciones de su lengua materna el niño primero aprende a imitar un patrón estructural, y luego aprende a combinar partes de otros patrones estructurales por analogía. Estos patrones se convierten en hábitos y al niño le facilita el aprendizaje el hecho de que ha comenzado con una mente en blanco; simplemente se le termina de llenar el pizarrón que antes tenía en blanco. Una consecuencia de esta teoría es el postulado de que a un niño no se le debería pedir que aprenda más de un idioma a la vez porque de lo contrario es muy posible que no aprenda ninguno de los dos a la perfección debido a la interferencia que cada idioma produce sobre el aprendizaje del otro. Otra consecuencia de la teoría es la idea de que a los adultos les cuesta aprender un segundo idioma porque para aprenderlo es necesario escribir en un pizarrón que ya está lleno. De acuerdo con esta teoría, el lenguaje es un conjunto de hábitos que ha adquirido el niño y los ha podido adquirir debido a su capacidad general para aprender. Es decir, el lenguaje forma parte íntegra de la conducta general del niño y no se diferencia en el fondo de la capacidad que tiene para aprender a manejar una bicicleta o un automóvil cuando sea más grande. Los lingüistas que siguen esta teoría se autodenominan 'estructuralistas'. Los estructuralistas creen que como el lenguaje no es más que la manifestación de una parte de una conducta general más amplia, también está bajo el control de estímulos, si bien el número de ellos es tal que la mente humana no los puede captar. El fundador del estructuralismo norteamericano, Leonard Bloomfield, expresó que si fuera posible conocer todos los estímulos a los cuales había estado sujeta una persona desde el momento de su concepción, o aún antes - todo cambio de temperatura, el cambio de posición de cada persona con quien había hablado, cada vez que había roncado y la duración de esto, etc. -sería posible predecir en un momento dado exactamente lo que esa persona diría o haría en el momento siguiente. El mismo Bloomfield reconoce que para poder hacer eso uno tendría que ser Dios, pero el hecho es que para Bloomfield el hombre no es más que una máquina sumamente complicada.

A partir de 1957 la mayoría de los lingüistas norteamericanos han rechazado esta teoría y han aceptado la teoría racionalista de Noam Chomsky. Si bien la teoría no tiene nada de nuevo en sí, pues existía antes de Aristóteles y era la teoría predominante desde Descartes hasta principios de este siglo, Chomsky ha logrado captar la imaginación tanto de filósofos y de psicólogos como de lingüistas por el rigor con que la ha elaborado y formalizado. Chomsky pretende buscar,evidencia entre los idiomas del mundo para demostrar que el hombre no es una máquina sino un creador. Pretende demostrar que el hombre a diferencia de los animales, no actúa bajo el control de estímulos y que 'la comunicación humana y la comunicación animal se unen únicamente en un nivel de generalidad que incluye también casi toda otra conducta?

Comienza Chomsky por notar la edad tan inmadura en que el niño aprende a hablar y la rapidez con que aprende. Ya a la edad de tres años, o poco más, la mayoría de los niños conocen y pueden reproducir una enorme cantidad de los patrones estructurales de su lengua materna, y antes de cumplir los seis años conocen casi todos. Esto sucede en un momento cuando la capacidad intelectual y la capacidad física del niño están en el punto mínimo de su desarrollo.

Chomsky hace notar que cuando el niño comienza a hablar no produce oraciones de estructura sencilla y limitada, sino que de una vez produce oraciones de una complejidad extraordinaria con un alto nivel de abstracción. El niño ni oye ni produce oraciones en forma escalonada u ordenada de manera secuencial. En lugar de aprender 
de manera inductiva -0 , sea por analogíatoda la evidencia está en que el niño aprende de manera deductiva $o$, sea, que forma reglas a través de la abstracción: está inmerso en el lenguaje, pero fuera de ciertos clichés sui géneris lo que oye son casi siempre oraciones completamente nuevas.

Si fuera posible comparar los billones de oraciones que se pronuncian todos los días, pronto nos daríamos cuenta de que la posibilidad de que una oración dada se repita es prácticamente cero. El niño puede aprender partes de estas oraciones, pero para saber cómo producir las suyas propias, cada una de las cuales a la vez va a ser casi única, tiene que haber deducido la estructura de la lengua que se está hablando a su alrededor. $\mathrm{Al}$ igual que el lingüista, el niño no puede observar esta estructura directamente. Solamente puede basar sus deducciones en la manifestación lingüística que oye, y gran parte de ésta está determinada por un sistema lingüístico abstracto que en el mejor de los casos se refleja de manera indirecta y con mucha imperfección -por ejemplo, hay frecuentes vacilaciones y arranques falsos en el habla. Dada la manifestación casi única de cada oración que se pronuncia, es mucho pedir que se crea que el niño aprende por imitación. Lo más asombroso que hay en el aprendizaje de un idioma es que llegue a producirse, dada la edad tan inmadura del aprendiz y el corto tiempo que se necesita para llevarse a cabo.

Chomsky ha señalado también lo parecidos que en el fondo son todos los idiomas del mundo que se han podido describir, quitado todo lo superficial que contienen: vocabularios diferentes, diferentes maneras de expresar redundancias, etc. Si nos fijamos en las relaciones que existen entre las diferentes clases de oraciones de los diversos idiomas del mundo, nos damos cuenta de lo parecidos que "son. Veamos un ejemplo concreto El español y el inglés se parecen mucho, cosa que por demás no sorprende puesto que tienen el mismo origen. Pero estos idiomas son muy diferentes del bribri, que se habla en el sur de Costa Rica. En español cuando decimo 'Juan está tomando cerveza', vemos que las ideas básicas que contiene esta oración son 'Juan, tomar, cerveza' y que está presente la idea de 'ahora mismo'. El hecho de que se diga 'está' y no 'estoy' o 'estamos' es superficial, como también lo es la selección de los rubros léxicos. Es interesante el hecho de que se diga 'tomando, pero la terminación '-ndo' obedece a una regla para el español que dice que después de 'estar' el verbo tiene que terminar así para expresar la idea de 'ahora mismo'. En inglés sucede una situación análoga en 'John is drinking beer'. Lo sorprendente es que sucede exactamente lo mismo en bribri: Juan tso' servésa džök (Juan está cerveza tomando). El morfema tso' corresponde a 'estar', y -ök corresponde exactamente a ' - ndo'. Si se compara esto con el alemán 'Johann trinkt Bier' o el ruso 'Ivan p'ot p'ivo', vemos que en estos idiomas falta la forma perifrástica. Pero en estos idiomas las oraciones resultan ambiguas y los hablantes de alemán o de ruso las pueden reconocer como tales e indicar en que consiste la ambigüedad.

$\mathrm{Si}$ en realidad todos los idiomas son tan parecidos en el fondo, quitadas las partes superficiales, según Chomsky la razón debe ser que por lo menos una parte del lenguaje mismo es innata, es decir es transmitida genéticamente. Para Chomsky el hecho de que no haya idioma en el mundo que no tenga una representación para 'frase nominal' y 'frase verbal' es indicio de que estas dos relaciones son innatas. Chomsky pretende afirmar que el niño tiene un mecanismo intrínseco que lo capacita para reconocer una relación de conformidad abstracta entre elementos y/o secuencias de elementos en su corpus, es decir el lenguaje que tiene a su disposición. Mediante su teoría de una gramática transformacional generativa, Chomsky trata de presentar en forma gráfica un modelo que caracteriza este mecanismo. Por ejemplo, se representa la oración 'Tú brincas' con una fórmula del tipo IXJ, en la cual $\mathrm{I}=$ 'tú', $\mathrm{X}=$ brinca, $\mathrm{y} \mathrm{J}={ }^{-}-\mathrm{s}$ '. El niño tiene la capacidad para abstraer (IJ) (X) de IXJ y aplicar la regla (IJ) (X) $\rightarrow$ IXJ cada vez que se reúnan las condiciones necesarias. Dada la información sintáctica del valor de los variables de la fórmula, el niño tiene la capacidad intrínseca de saber que 'Tú brincas' (tú-s + brinca) es un ejemplo de la regla, pero que 'El y tú brincan' no lo es (el español no permite la secuencia * 'El y tú 
brincas').

Otro caso que tiende a demostrar la capacidad de abstracción que tiene el niño es el siguiente: en inglés parece ser que hay una etapa en el aprendizaje del idioma en que al niño le presentan dificultades los grupos consonánticos iniciales que comienzan con 's', de modo que durante cierto tiempo pronuncia estas palabras sin la 's' inicial. Como resultado, las palabras 'cool' y 'school' se pronuncian como homófonos. Lo curioso del caso es que cuando el niño comienza a pronunciar la 's' inicial la coloca exactamente en el lugar donde debe ir en todos los casos, sin un solo error, y nunca la coloca en los lugares en donde no debe ir. Esto indica que la representación correcta de la pronunciación de estas palabras estaba presente en la mente del niño aún en la etapa cuando no se reflejaba en su habla.

La posición empírica y la posición racionalista se oponen en cuanto a la cantidad de estructura lingüística que se aprende, pero no están diametralmente opuestas. Los empiristas reconocen que la estructura del organismo humano coloca ciertas restricciones sobre la clase de sistema lingüístico que el humano puede llegar a dominar. Reconocen que cualquier sistema resultante es necesariamente finito porque el humano no es capaz de aprender un número infinito de reglas. Los empiristas aceptan, además, que el niño nace con alguna capacidad transmitida genéticamente que le permite combinar estructuras psicológicas sencillas para formar estructuras más complejas. Esta capacidad primitiva para aprender, por lo general, se concibe como una capacidad relativamente simple, algo parecido a la capacidad de formar asociaciones -como, por ejemplo, la asociación que existe entre los confites y el concepto de lo dulce. Puesto que en nuestra experiencia las dos cosas a menudo se dan juntas, cada una trae a la mente la otra.

Por lo tanto, la posición empirista acepta que las propiedades innatas del organismo son, hasta cierto punto, responsables de la determinación de la organización del lenguaje. $\mathrm{Al}$ mismo tiempo, los racionalistas no niegan que el aprendizaje juega un papel significativo en la adquisición del lenguaje. Lo que el niño hereda genéticamente no es la lengua específica, sino una capacidad para el lenguaje. Cuando nace, posee ya todas las propiedades de estructura y organización que comparten todos los idiomas, pero estas estructuras no son exhaustivas de la estructura de ningún idioma en particular. El sistema que se hereda es como un esqueleto que sirve de base para que el niño descubra aquellos detalles estructurales que la traduzcan al sistema lingüístico plenamente especificado que se emplea alrededor de él. El aprendizaje entra aquí como un factor importante porque el niño debe descubrir por sí mismo qué es lo que diferencia el idioma que está aprendiendo de la estructura general de todos los idiomas posibles. Pero este proceso de descubrimiento se realiza dentro de los límites impuestos por su herencia genética, y el papel que tiene el aprendizaje es por lo tanto mínimo.

Hasta aquí la diferencia entre la posición empírica y la posición racionalista es cuantitativa, y por lo tanto una diferencia de grado. Pero hay otra diferencia que es absoluta y que hace irreconciliables las dos posiciones. Sostienen los empiristas que el niño nace con una capacidad general para aprender, pero sin ninguna capacidad especial para el lenguaje. El niño construye un sistema lingüístico por medio de la experiencia usando la misma capacidad. intelectual básica -como la asociación, por ejemploque es responsable de la formación de todos los demás aspectos de su estructura cognocitiva.

Para los racionalistas, en cambio, además de una inteligencia general hay una capacidad innata especial para el lenguaje y ofrecen argumentos a favor de su posición. El primer argumento es la uniformidad con que toda la raza humana adquiere una lengua. Aún cuando haya toda clase de habilidades físicas e intelectuales que el niño no logra adquirir a pesar de los esfuerzos hechos para enseñárselas, todo niño aprende un idioma a menos que sea víctima de una defiencia mental en extremo grave o que esté aislado por completo del uso de algún idioma. Esto es exactamente lo que supone una hipótesis que considera que el lenguaje está especificado casi por completo de manera innata, y que la experiencia lingüística sirve principalmente para activar un sistema 
que se ha especificado genéticamente.

Esta uniformidad de adquisición lingüística choca con la posición empirista. $\mathrm{Si}$ la adquisición de una lengua dependiera del entrenamiento que recibe el niño, debería existir una correlación entre el entrenamiento recibido y las diferencias en el dominio de la lengua, si se mantiene constante la inteligencia en general. Pero de hecho esto no ocurre. Todo niño aprende a hablar sea o no que alguien trate de ayudarle, que lo corrija o que lo ponga a hablar. A pesar de las grandes variaciones en cuanto a la cantidad de lenguaje a que está expuesto, todo niño adquiere un sistema lingüístico completo. Que se sepa, no hay ningún caso de un niño que haya aprendido la mitad de un idioma, que no tenga representaciones fonológicas latentes o que no sepa rubros lexicológicos. Las vicisitudes de su experiencia lingüística en su edad más temprana no se reflejan en posteriores variaciones comparables de estructura lingüística.

El segundo argumento, que ya se ha mencionado, es que únicamente la especie humana aprende a hablar. El chimpancé es anatómicamente similar al hombre y aprende a usar herramientas y a resolver problemas sencillos. La diferencia intelectual entre estos animales y el hombre, por lo tanto, no es una diferencia absoluta sino de grado. Pero el chimpancé nunca aprende a hablar, y es patente que el lenguaje no está directamente vinculado a la inteligencia.

El tercer argumento a favor de la especificación innata es la relativa perfección con que se adquiere un idioma. Si el lenguaje fuera un reflejo fiel de una inteligencia general y no de una capacidad lingüística específica, debería de existir una correlación entre diferencias de inteligencia y diferencias en la adquisición de un idioma si se mantuviera constante el entrenamiento. Sería de esperar que un niño inteligente llegara a dominar mejor un sistema lingüístico que el niño menos inteligente. Para algunos niños el aprendizaje de una lengua debería resultar en un completo fracaso, así como algunos niños nunca aprenden el procedimiento para sacar raíces cuadradas. No sería extraño que algu- nos niños terminaran con sistemas lingüísticos tan deficientes o tan deformes que serían irreconocibles. Pero nada de esto sucede. Absolutamente todos los niños aprenden a hablar, niños inteligentes y niños no inteligentes. Todos los niños logran dominar un sistema lingüístico que es prácticamente idéntico al sistema que sirve de modelo. Puede haber diferencias de poca importancia, tales como el tamaño de su vocabulario, pero no hay diferencia apreciable en cuanto a lo que es verdaderamente importante: los rasgos estructurales de la organización lingüística. Si como pretende la teoría racionalista el papel que juega el aprendizaje es mínimo, es imposible que surjan errores estructurales radicales.

Otro argumento a favor de la posición es la abstracción y la complejidad estructural de los idiomas. Durante siglos se ha estado estudiando el lenguaje en general y los idiomas en particular. A pesar de esto no se ha hecho un estudio exhaustivo de ningún idioma del mundo. Pero esto en efecto es lo que hace el niño al lograr dominar, además del vocabulario, todos los principios estructurales que constituyen un sistema lingüístico y en momentos cuando todavía no es capaz de pensar en forma lógica ni en forma analítica. Esto se explica fácilmente si la estructura lingüística es transmitida genéticamente. La tarea de aprendizaje que tiene el niño es la labor relativamente simple de rechazar todas las posibilidades innatas que no le sirvan, dejándose únicamente aquellas que sí le sirven.

Para los empiristas todo este sistema lingüístico se crea de la nada sobre la base de una capacidad intelectual general. Se pretende que el niño descubra, no unos detalles de estructura, sino toda la organización del sistema. Habiendo nacido sin expectaciones lingüísticas, se supone que en una edad preintelectual el niño haya hecho una serie de descubrimientos lingüísticos que en mucho sobrepasan los resultados acumulados de los esfuerzos de todos los eruditos que durante siglos han investigado el lenguaje. Esto simplemente no parece razonable. 


\section{BIBLIOGRAFIA}

Bloomfield, Leonard, 1933. Language.Nueva York: Holt.

Chomsky, Noam. 1957. Syntactic structures. La Haya: Mouton.

1965. Aspects of the theory of syntax. Cambridge, Mass; MIT Press.

1967. Some general properties of phonological rules. Language 43.102-128.

Chomsky, Noam y M. Halle. 1965. Some controversial questions in phonological theory Joumal of Linguistics. 1, 97-138.

Fodor, Jerry A. How to leran to talk: some simple ways. Trabajo poligrafiado.

Green, Judith. Rationalists and empiricists Trabajo poligrafiado.

Langeaker, Ronald W. 1968. Language and its structure. Nueva York, Harcourt.

Miller, Wick and Susan Ewin, Noam Chomsky. Formal discussion of "the development of grammar in child language. Trabajo poligrafiado.

Wilson, Jack. 1972. Metameta teoria de lenguaje. San José: Revista de la Universidad de Costa Rica.

1974. Structuralism. San José: Revista de la Universidad de Costa Rica. 